\title{
Genetic analysis of subsequent second primary malignant neoplasms in long-term pancreatic cancer survivors suggests new potential hereditary genetic alterations
}

This article was published in the following Dove Press journal: Cancer Management and Research

\begin{abstract}
Martin Lovecek, ${ }^{1, *}$ Marketa Janatova, ${ }^{2, *}$ Pavel Skalicky, ' Tomas Zemanek, ${ }^{3}$ Roman Havlik, 'Jiri Ehrmann, ${ }^{4}$ Ondrej Strouhal, ${ }^{3}$ Petra Zemankova, ${ }^{2}$ Klara Lhotova, ${ }^{2}$ Marianna Borecka, ${ }^{2}$ Jana Soukupova, ${ }^{2}$ Hana Svebisova, ${ }^{3}$ Pavel Soucek, ${ }^{5}$ Viktor Hlavac, ${ }^{5}$ Zdenek Kleibl, ${ }^{2}$ Cestmir Neoral,' Bohuslav Melichar, ${ }^{3}$ Beatrice Mohelnikova-Duchonova ${ }^{3}$

'Department of Surgery I, Faculty of Medicine and Dentistry, Palacky University Olomouc, University Hospital Olomouc, Olomouc, Czech Republic; ${ }^{2}$ Institute of Biochemistry and Experimental Oncology, First Faculty of Medicine, Charles University, Prague, Czech Republic; ${ }^{3}$ Department of Oncology, Institute of Molecular and Translational Medicine, Faculty of Medicine and Dentistry, Palacky University, Olomouc, Czech Republic; ${ }^{4}$ Department of Clinical and Molecular Pathology, Faculty of Medicine and Dentistry, Palacky University Olomouc, University Hospital Olomouc, Olomouc, Czech Republic; ${ }^{5}$ Biomedical Center, Faculty of Medicine in Pilsen, Charles University, Pilsen Czech Republic

*These authors contributed equally to this work
\end{abstract}

Correspondence: Beatrice

Mohelnikova-Duchonova

Department of Oncology, Institute of Molecular and Translational Medicine, Faculty of Medicine and Dentistry, Palacky University Olomouc, University Hospital Olomouc, I.P. Pavlova 6, 77900 Olomouc, Czech Republic

Tel +420775270283

Email d.beatrice@seznam.cz
Background: The principal aim of this report was to study second primary malignant neoplasms (SMNs) in long-term survivors of pancreatic ductal adenocarcinoma (PDAC) with regard to the germline genetic background.

Patients and methods: A total of 118 PDAC patients after a curative-intent surgery who were treated between 2006 and 2011 were analyzed. Of the 22 patients surviving for $>5$ years, six went on to develop SMNs. A genetic analysis of 219 hereditary cancer-predisposition and candidate genes was performed by targeted next-generation sequencing in germline DNA from 20 of these patients.

Results: Of all the radically resected PDAC patients, six patients went on to subsequently develop SMNs, which accounted for $27 \%$ of the long-term survivors. The median time to diagnosis of SMNs, which included two cases of rectal cancer, and one case each of prostate cancer, malignant melanoma, breast cancer, and urinary bladder cancer, was 52.5 months. At the time of analysis, none of these patients had died as a result of PDAC progression. We identified four carriers of germline pathogenic mutations in 20 analyzed long-term survivors. One carrier of the CHEK2 mutation was found among four analyzed patients who developed SMNs. Of the remaining 16 long-term PDAC survivors, 3 patients (19\%) carried germline mutation(s) in the $M L H 1+A T M, C H E K 2$, and RAD51D gene, respectively.

Conclusion: This retrospective analysis indicates that SMNs in PDAC survivors are an important clinical problem and may be more common than has been acknowledged to be the case. In patients with good performance status, surgical therapy should be considered, as the SMNs often have a favorable prognosis.

Keywords: pancreatic ductal adenocarcinoma, second primary neoplasms, subsequent malignant neoplasm, hereditary cancer genes, long-term survivors, surgical treatment

\section{Introduction}

Pancreatic ductal adenocarcinoma (PDAC) is a malignant tumor with an extremely poor prognosis. Among radically operated patients in high-volume centers, five-year survival rates are as low as 4\%-34\%, with a median survival ranging between 17 and 27 months. $^{1}$

Subsequent second primary malignant neoplasm (SMN) is a term used to describe a new primary cancer that occurs in a patient who has been diagnosed and treated for cancer in the past, months or years after the original primary cancer. SMNs are a major cause of mortality and serious morbidity among cancer survivors who have been 
successfully cured of their first cancer. Their etiologies are multiple and may relate to the role of primary cancer treatment (mainly radiotherapy and chemotherapy), unhealthy lifestyle behaviors, germline and somatic mutations, aging, and most likely a combination of any of these factors., ${ }^{2,3}$ Because of the unfavorable prognosis, very few long-term PDAC survivors will develop SMN., ${ }^{2,3}$ Consequently, there are very few reports about SMNs in PDAC survivors and their prognosis, and there is no information on the genetic background of these patients. ${ }^{2-9}$

The aim of the present study was to identify and describe SMNs in long-term PDAC survivors with regard to their potential genetic background. This is the first study describing the genetic background of long-term PDAC survivors with SMNs.

\section{Patients and methods}

\section{Patients}

This retrospective study involved 118 Caucasian patients with PDAC, who had undergone a curative-intent surgery between 2006 and 2011 at the University Hospital, Olomouc, Czech Republic.

The inclusion criteria for further SMN analysis included a curative-intent surgical treatment, histologic diagnosis of PDAC independently confirmed by two experienced pathologists, at least a five-year survival period after surgery, and postresection follow-up comprising biochemical tumor marker monitoring (CA 19-9, CEA, and CA 125) every 3 months and imaging (computed tomography $[\mathrm{CT}]$ or positron emission tomography [PET]/CT) scans performed every 6-12 months or in the case of CA 19-9 elevation.

The clinical data, including age, gender, date of diagnosis, pTNM stage, ${ }^{10}$ the histologic type and grade of the tumor, lymphatic, vascular, and perineural invasion, the therapy administered and follow-up, were obtained from medical records. The main clinical characteristics of the whole group are summarized in Table 1. The retrospective study was approved by the Institutional Review Board of the University Hospital in Olomouc, and all living patients gave their informed written consent to participation in the study and the genetic analysis. The study was conducted in accordance with the Declaration of Helsinki.

The principal objective of this study was the identification of SMNs in this cohort of patients. The criteria used for the definition of SMN were derived from Waren and Gates, including a histologic confirmation of the second primary malignancy, anatomical separations of both tumors or recurrence exclusion, and a second tumor diagnosis $>6$
Table I Baseline patient characteristics (entire cohort)

\begin{tabular}{|c|c|c|}
\hline Parameters & Number of patients* & $\%$ \\
\hline \multicolumn{3}{|l|}{ Sex } \\
\hline Male & 75 & 64 \\
\hline Female & 43 & 36 \\
\hline \multicolumn{3}{|l|}{ TNM stage } \\
\hline 1 & 20 & 17 \\
\hline IIA & 34 & 29 \\
\hline IIB & 54 & 46 \\
\hline III & 2 & 2 \\
\hline IV & 8 & 7 \\
\hline \multicolumn{3}{|l|}{ Histologic grade } \\
\hline GI + G2 (well to moderate) & 62 & 52 \\
\hline G3 (poor) & 51 & 44 \\
\hline Not available & 5 & 4 \\
\hline \multicolumn{3}{|l|}{ Lymphovascular invasion } \\
\hline$p L 0$ & 74 & 63 \\
\hline $\mathrm{pLI}$ & 38 & 32 \\
\hline Not available & 6 & 5 \\
\hline \multicolumn{3}{|l|}{ Perineural invasion } \\
\hline pPO & 35 & 30 \\
\hline $\mathrm{pPI}$ & 77 & 65 \\
\hline Not available & 6 & 5 \\
\hline \multicolumn{3}{|l|}{ Angioinvasion } \\
\hline pA0 & 91 & 77 \\
\hline $\mathrm{pAI}$ & 21 & 18 \\
\hline Not assessed & 6 & 5 \\
\hline \multicolumn{3}{|l|}{ Adjuvant therapy } \\
\hline Yes & 79 & 68 \\
\hline No & 37 & 31 \\
\hline Unknown & 2 & 2 \\
\hline
\end{tabular}

Note: *I 18 patients in total.

months after the diagnosis of the first tumor. ${ }^{2}$ The SMNs in the studied cohort were diagnosed by physical examination, endoscopy, and/or diagnostic imaging (CT/PET-CT) and were histologically verified.

\section{Next-generation sequencing analysis}

Blood was collected during diagnostic procedures using tubes with $\mathrm{K}_{3}$ EDTA anticoagulant, and DNA was isolated from lymphocytes using the phenol/chloroform extraction method described by Sugimura. ${ }^{11}$

A custom-designed CZECANCA panel (SeqCap EZ choice; Nimblegen/Roche) for the germline-targeted nextgeneration sequencing (NGS) analysis of cancer-predisposition and candidate genes was used as described previously. ${ }^{12}$ In brief, the panel targets 219 selected genes with a known predisposition to hereditary cancer syndromes (including breast, ovarian, colorectal, pancreatic, gastric, endometrial, kidney, prostate, and skin cancers) and other genes that code for proteins involved in the DNA repair and/or DNA damage response with uncertain clinical relevance. A sequencing 
library was prepared using the KAPA HTP Library Preparation kit according to the manufacturer's instructions (KAPA Biosystems, Roche) and sequenced on the MiSeq instrument with MiSeq reagent Kit v3 (Illumina).

\section{Bioinformatics analysis}

The NGS data were processed according to the in-house bioinformatics pipeline as described recently. ${ }^{12}$ In brief, SAM files were generated from FASTQ files using Novoalign v2.08.03 and transformed into BAM files using Picard tools v1.129. The VCF files prepared by GATK were annotated by ANNOVAR. ${ }^{13}$ Medium-size indel identification was based on the method of soft-clipped bases using Pindel software, and copy number variation (CNV) analysis was performed using CNV kit. During variant filtration, we excluded low-quality variants (sequence quality $<30$ ) and common variants with allelic frequencies $>0.01$ in ESP6500 and 1,000 genomes databases, respectively. We also excluded variants present $>2 \times$ in a national database of genotypes that included 507 noncancer controls (data not shown). Nonsense, frameshift, and consensus dinucleotide splice site variants $( \pm 1 / 2)$ in known predisposition genes were classified as pathogenic or likely pathogenic. Missense variants, silent variants, inframe indels, and other intronic variants were considered only when reaching a CADD score $>2$ and gerp $>0$ and classified according to the ClinVar and/or VarSome database. Prioritized variants were further analyzed by three prediction tools (SIFT, PolyPhen-2, and Mutation Analyzer). Variants predicted to be damaging by at least two programs were considered potentially deleterious.

\section{Results}

\section{Patients and treatment}

Twenty-two patients (19.1\%) with histopathologically verified PDAC survived for $>5$ years since the primary PDAC diagnosis (long-term survivors) and matched the inclusion criteria for this retrospective study. The median follow-up was 6.2 years (range 5-11 years). Long-term PDAC survivors were further screened for the development of SMNs.

Overall, six patients (5.1\% of all radically resected PDAC patients) developed SMNs. The SMN rate among long-term survivors was $27 \%(\mathrm{~N}=6 / 22)$. The mean age of the long-term PDAC survivors at the time of PDAC diagnosis was $61.7 \pm 7.8$ years (range 44-75 years). The subgroup of patients with SMNs consisted of five males and only one female; the mean age was $66.7 \pm 7.4$ years (range $51-75$ years) at the time of PDAC diagnosis. None of these patients received neoadjuvant chemotherapy. One patient was treated with chemotherapy based on 5 -fluorouracil $\left(300 \mathrm{mg} / \mathrm{m}^{2} /\right.$ day) concomitant to radiotherapy (50.4 Gy in 5.5 weeks) in the adjuvant setting, and the other five patients were treated with six 4-week cycles of gemcitabine $\left(1000 \mathrm{mg} / \mathrm{m}^{2}\right.$ at days 1,8 , and 22$)$. Overall, of the long-term PDAC survivors in the present cohort, around $40 \%$ of patients who received gemcitabine postoperatively developed subsequent malignant neoplasms. The clinical and pathologic data of the patients with SMN are summarized in Table 2 .

\section{Timing and patterns of subsequent secondary malignant neoplasms}

The median time to SMN was 52.5 months (range 8.8-87.1 months; Table 2). The SMNs observed included two cases of rectal cancer, and one case each of prostate cancer, malignant melanoma, breast cancer, and urinary bladder cancer. Four of these patients underwent a curative surgery for the SMN. The patient with urinary bladder cancer underwent a radical cystectomy 63 months after PDAC resection. The patient with malignant melanoma underwent a radical excision 45.4 months after PDAC resection, and the patient with breast cancer underwent mastectomy 8.8 months after PDAC resection. All these patients are still alive with no recurrence of primary or secondary malignancy (6.3-8.9 years following the primary surgery of PDAC). One patient with rectal cancer died of postoperative complications from rectal surgery 64 months after the PDAC surgery. A second patient with rectal cancer died of cardiovascular comorbidities 62 months after the PDAC surgery without a specific therapy.

Prostate cancer with bone metastases was diagnosed in one patient 87.1 months after the primary PDAC resection and the patient was treated with hormonal therapy.

In summary, none of these patients died as a result of the PDAC.

\section{Genetic analysis}

A targeted NGS analysis covering 219 PDAC and other cancer susceptibility genes (Table 3) was performed in 20 patients both with and without SMNs (DNA samples from the two deceased patients with rectal cancer were not available).

Deleterious germline mutations were identified in 4 out of 20 NGS-analyzed long-term survivors (20\%; Table 4). One patient harbored two deleterious mutations (in $M L H 1$ and $A T M$ ). Of the four sequenced long-term survivors who developed SMN, one female patient who developed breast cancer 1 year after primary PDAC diagnosis with no family cancer history carried a deleterious missense mutation in CHEK2 (c.349A > G, p.Arg117Gly). Two out of 3 carriers of a 


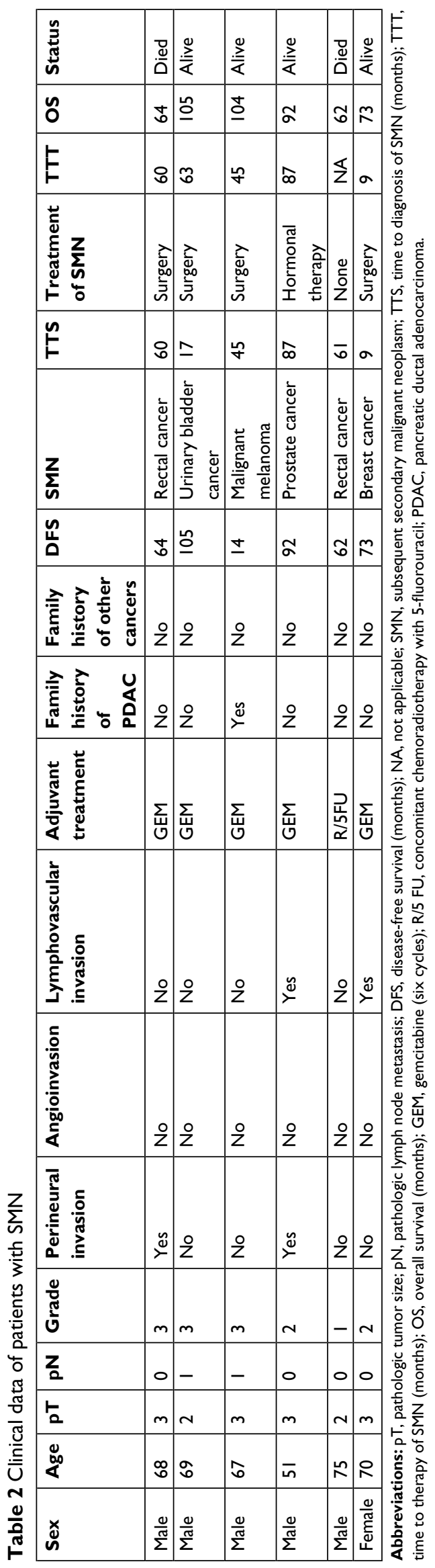

pathogenic mutation in 16 long-term PDAC survivors without SMN had a positive family cancer history. A patient with $R A D 51 D$ splice-site mutation c. $345+2 \mathrm{~T}>\mathrm{G}$ had a mother with gastric cancer and a patient with two mutations (nonsense variant in $M L H 1$ : c. $390 \mathrm{C}>\mathrm{G}$ and frame-shift variant in ATM: c.3849delA) had a father with a colorectal cancer and a father's mother with brain tumor. The remaining patient with the CHEK2 c.1100delC mutation had no personal or family cancer history.

Subsequently, we identified several alterations with unknown impact on protein function. Fourteen variants in ten patients were predicted to be damaging by at least three prediction programs (Table 5).

\section{Discussion}

This report demonstrates a relatively high incidence of SMNs in five-year survivors of PDAC. The incidence of SMNs is generally $2 \%-10 \%$ and the prevalence is $6.6 \%-9 \%$, accounting for about $16 \%$ of overall cancer incidence. ${ }^{2,3,5}$ So far, very few publications have reported an analysis of second primary extrapancreatic malignancies following PDAC, probably because of the poor prognosis of these patients. ${ }^{2,6-9}$ A large population-based study calculated the incidence of SMNs diagnosed after the diagnosis of PDAC to be lower when compared to other cancers (around 1.3\%). ${ }^{8,14}$ The latest report of the Czech National Cancer Registry shows a primary PDAC incidence of about $84 \%$ and a second primary PDAC (PDAC as the second primary tumor) incidence of about $16 \%$. The incidence of synchronous PDAC and other malignancies is $5 \%$ of total PDAC patient incidence and the incidence of SMNs following PDAC is $<1 \%$ of the total. ${ }^{15}$ These rates were confirmed by the study reported by Hackert et al. ${ }^{16}$

The unexpectedly high number of SMNs $(5 \%)$ in the present cohort of resected PDAC patients may be primarily explained by the comprehensive follow-up focusing not only on PDAC recurrence, but also on SMNs. Moreover, among long-term PDAC survivors, we identified SMNs in $27 \%$ of patients, indicating that the apparently limited number of SMNs in PDAC reported so far may be largely due to the poor prognosis. Previously published reports on long-term PDAC survivors show prevalences of SMNs ranging between $0 \%$ and $20 \%$. ${ }^{6,7}$ Nevertheless, this retrospective analysis may indicate that the development of SMNs in PDAC survivors may be more frequent than has been acknowledged in previous reports.

Improved medical options including anticancer therapy and treatment individualization lead to the prolongation of survival. This is evident in survivors of various primary 
Table 3 List of genes analyzed by targeted next-generation sequencing

\begin{tabular}{|c|c|}
\hline Abbreviation & Gene name (alternative denominations) \\
\hline AlP & Aryl hydrocarbon receptor interacting protein \\
\hline ALK & Anaplastic lymphoma kinase \\
\hline APC & Adenomatous polyposis coli \\
\hline APEXI & APEX nuclease (multifunctional DNA repair enzyme) I \\
\hline ATM & Ataxia telangiectasia mutated \\
\hline ATMIN & ATM interactor \\
\hline ATR & Ataxia telangiectasia and $\mathrm{Rad} 3$ related \\
\hline ATRIP & ATR interacting protein \\
\hline AURKA & Aurora kinase $\mathrm{A}$ \\
\hline AXINI & Axin I \\
\hline BABAMI & BRISC and BRCAI A complex member I \\
\hline BAPI & BRCAI-associated protein-I (ubiquitin carboxy-terminal hydrolase) \\
\hline BARDI & BRCAI-associated RING domain I \\
\hline BLM & Bloom syndrome, RecQ helicase-like \\
\hline$B M P R I A$ & Bone morphogenetic protein receptor, type IA \\
\hline BRAP & BRCAI-associated protein \\
\hline BRCAI & Breast cancer I, early onset \\
\hline BRCA2 & Breast cancer 2, early onset \\
\hline BRCC3 & BRCAI/BRCA2-containing complex, subunit 3 \\
\hline BRE & Brain and reproductive organ-expressed (TNFRSFIA modulator) \\
\hline BRIPI & BRCAI interacting protein C-terminal helicase I \\
\hline$B U B / B$ & Budding uninhibited by benzimidazoles I homolog beta (yeast) \\
\hline $\mathrm{Cl}$ lorf30 & Chromosome II open reading frame 30 (EMSY) \\
\hline Cl9orf40 & Chromosome 19 open reading frame 40 (FAAP24) \\
\hline CASP8 & Caspase 8, apoptosis-related cysteine peptidase \\
\hline CCNDI & Cyclin DI \\
\hline CDC73 & Cell division cycle 73, PafI/RNA polymerase II complex component, homolog (Saccharomyces cerevisiae) \\
\hline $\mathrm{CDHI}$ & Cadherin I, type I, E-cadherin (epithelial) \\
\hline CDK4 & Cyclin-dependent kinase 4 \\
\hline$C D K N / B$ & Cyclin-dependent kinase inhibitor IB (p27, KipI) \\
\hline CDKNIC & Cyclin-dependent kinase inhibitor IC (p57, Kip2) \\
\hline CDKN2A & Cyclin-dependent kinase inhibitor $2 \mathrm{~A}$ \\
\hline CEBPA & CCAAT/enhancer binding protein (C/EBP), alpha \\
\hline CEP57 & Centrosomal protein $57 \mathrm{kDa}$ \\
\hline CLSPN & Claspin \\
\hline CSNKID & Casein kinase I, delta \\
\hline CSNKIE & Casein kinase I, epsilon \\
\hline CWFI9L2 & CWFI9-like 2, cell cycle control (Schizosaccharomyces pombe) \\
\hline CYLD & Cylindromatosis (turban tumor syndrome) \\
\hline DCLREIC & DNA cross-link repair IC \\
\hline DDB2 & Damage-specific DNA binding protein 2, $48 \mathrm{kDa}$ \\
\hline DHFR & Dihydrofolate reductase \\
\hline DICERI & Dicer I, ribonuclease type III \\
\hline DMCI & DMCI dosage suppressor of mckI homolog, meiosis-specific homologous recombination (yeast) \\
\hline DNAJC2I & DnaJ (Hsp40) homolog, subfamily C, member 21 \\
\hline DPYD & Dihydropyrimidine dehydrogenase \\
\hline EGFR & Epidermal growth factor receptor \\
\hline EPCAM & Epithelial cell adhesion molecule \\
\hline EPHXI & Epoxide hydrolase I, microsomal (xenobiotic) \\
\hline ERCCI & Excision repair cross-complementing rodent repair deficiency, complementation group I \\
\hline ERCC2 & Excision repair cross-complementing rodent repair deficiency, complementation group 2 \\
\hline ERCC3 & Excision repair cross-complementing rodent repair deficiency, complementation group 3 \\
\hline ERCC4 & Excision repair cross-complementing rodent repair deficiency, complementation group 4 \\
\hline ERCC5 & Excision repair cross-complementing rodent repair deficiency, complementation group 5 \\
\hline ERCC6 & Excision repair cross-complementing rodent repair deficiency, complementation group 6 \\
\hline ESRI & Estrogen receptor I \\
\hline
\end{tabular}


Table 3 (Continued)

\begin{tabular}{|c|c|}
\hline Abbreviation & Gene name (alternative denominations) \\
\hline ESR2 & Estrogen receptor 2 (ER beta) \\
\hline EXOI & Exonuclease I \\
\hline EXTI & Exostosin I \\
\hline EXT2 & Exostosin 2 \\
\hline EYA2 & Eyes absent homolog 2 (Drosophila) \\
\hline$E Z H 2$ & Enhancer of zeste homolog 2 (Drosophila) \\
\hline FAM I 75A & Family with sequence similarity 175, member $\mathrm{A}$ \\
\hline FAM I 75B & Family with sequence similarity 175, member B \\
\hline FANI & FANCD2/FANCl-associated nuclease I \\
\hline FANCA & Fanconi anemia, complementation group $\mathrm{A}$ \\
\hline FANCB & Fanconi anemia, complementation group B \\
\hline FANCC & Fanconi anemia, complementation group C \\
\hline FANCD2 & Fanconi anemia, complementation group D2 \\
\hline FANCE & Fanconi anemia, complementation group $\mathrm{E}$ \\
\hline FANCF & Fanconi anemia, complementation group $\mathrm{F}$ \\
\hline FANCG & Fanconi anemia, complementation group G \\
\hline FANCl & Fanconi anemia, complementation group I \\
\hline FANCL & Fanconi anemia, complementation group L \\
\hline FANCM & Fanconi anemia, complementation group $M$ \\
\hline FBXW7 & F-box and WD repeat domain containing 7, E3 ubiquitin protein ligase \\
\hline $\mathrm{FH}$ & Fumarate hydratase \\
\hline FLCN & Folliculin \\
\hline GADD45A & Growth arrest and DNA-damage-inducible, alpha \\
\hline GATA2 & GATA binding protein 2 \\
\hline GPC3 & Glypican 3 \\
\hline GRB7 & Growth factor receptor-bound protein 7 \\
\hline HELQ & Helicase, POLQ-like \\
\hline HNFIA & HNFI homeobox A \\
\hline$H O X B / 3$ & Homeobox $\mathrm{Bl} 3$ \\
\hline HRAS & v-Ha-ras Harvey rat sarcoma viral oncogene homolog \\
\hline HUSI & HUSI checkpoint homolog (S. pombe) \\
\hline CHEKI & Checkpoint kinase I \\
\hline CHEK2 & Checkpoint kinase 2 \\
\hline KAT5 & K(lysine) acetyltransferase 5 \\
\hline KCNJ5 & Potassium inwardly rectifying channel, subfamily J, member 5 \\
\hline KIT & V-kit Hardy-Zuckerman 4 feline sarcoma viral oncogene homolog \\
\hline LIGI & Ligase I, DNA, ATP-dependent \\
\hline LIG3 & Ligase III, DNA, ATP-dependent \\
\hline LIG4 & Ligase IV, DNA, ATP-dependent \\
\hline LMOI & LIM domain only I (rhombotin I) \\
\hline LRIGI & Leucine-rich repeats and immunoglobulin-like domains I \\
\hline MAX & MYC-associated factor $X$ \\
\hline $\mathrm{MCPHI}$ & Microcephalin I \\
\hline $\mathrm{MDCl}$ & Mediator of DNA-damage checkpoint I \\
\hline MDM2 & Mdm2, p53 E3 ubiquitin protein ligase homolog (mouse) \\
\hline MDM4 & Mdm4 p53 binding protein homolog (mouse) \\
\hline MENI & Multiple endocrine neoplasia I \\
\hline MET & Met proto-oncogene (hepatocyte growth factor receptor) \\
\hline MGMT & O-6-methylguanine-DNA methyltransferase \\
\hline MLHI & mutL homolog I, colon cancer, nonpolyposis type 2 (Escherichia coli) \\
\hline MLH3 & mutL homolog 3 (E. coli) \\
\hline MMP8 & Matrix metallopeptidase 8 (neutrophil collagenase) \\
\hline MPL & Myeloproliferative leukemia virus oncogene \\
\hline MREIIA & MREII meiotic recombination II homolog A (S. cerevisiae) \\
\hline MSH2 & mutS homolog 2, colon cancer, nonpolyposis type I (E. coli) \\
\hline MSH3 & mutS homolog 3 (E. coli) \\
\hline
\end{tabular}


Table 3 (Continued)

\begin{tabular}{|c|c|}
\hline Abbreviation & Gene name (alternative denominations) \\
\hline MSH5 & mutS homolog 5 (E. coli) \\
\hline MSH6 & mutS homolog 6 (E. coli) \\
\hline MSRI & Macrophage scavenger receptor I \\
\hline MUS8I & MUS8I endonuclease homolog (S. cerevisiae) \\
\hline MUTYH & mutY homolog (E. coli) \\
\hline NATI & $\mathrm{N}$-acetyltransferase I (arylamine $\mathrm{N}$-acetyltransferase) \\
\hline NBN & Nibrin \\
\hline NCAMI & Neural cell adhesion molecule I \\
\hline NELFB & Cofactor of BRCAI \\
\hline NFI & Neurofibromin I \\
\hline NF2 & Neurofibromin 2 (merlin) \\
\hline NFKBIZ & Nuclear factor of kappa light polypeptide gene enhancer in B-cells inhibitor, zeta \\
\hline NHEJI & Nonhomologous end-joining factor I \\
\hline NSDI & Nuclear receptor binding SET domain protein I \\
\hline OGGI & 8-oxoguanine DNA glycosylase \\
\hline PALB2 & Partner and localizer of BRCA2 \\
\hline PARPI & Poly (ADP-ribose) polymerase I \\
\hline PCNA & Proliferating cell nuclear antigen \\
\hline PHB & Prohibitin \\
\hline PHOX2B & Paired-like homeobox $2 b$ \\
\hline PIK3CG & Phosphatidylinositol-4,5-bisphosphate 3-kinase, catalytic subunit gamma \\
\hline PLA2G2A & Phospholipase A2, group IIA (platelets, synovial fluid) \\
\hline PMSI & PMSI postmeiotic segregation increased I (S. cerevisiae) \\
\hline$P O L B$ & Polymerase (DNA directed), beta \\
\hline POLDI & Polymerase (DNA directed), delta I, catalytic subunit \\
\hline POLE & Polymerase (DNA directed), epsilon, catalytic subunit \\
\hline PPMID & Protein phosphatase, $\mathrm{Mg} 2+/ \mathrm{Mn} 2+$ dependent, ID \\
\hline PREX2 & Phosphatidylinositol-3,4,5-trisphosphate-dependent Rac exchange factor 2 \\
\hline PRFI & Perforin I (pore forming protein) \\
\hline PRKARIA & Protein kinase, cAMP-dependent, regulatory, type I, alpha \\
\hline PRKDC & Protein kinase, DNA-activated, catalytic polypeptide \\
\hline PTEN & Phosphatase and tensin homolog \\
\hline PTCHI & Patched I \\
\hline PTTG2 & Pituitary tumor-transforming 2 \\
\hline RADI & RADI homolog (S. pombe) \\
\hline$R A D / 7$ & RADI7 homolog (S. pombe) \\
\hline RAD/8 & RADI8 homolog (S. cerevisiae) \\
\hline RAD23B & RAD23 homolog B (S. cerevisiae) \\
\hline RAD50 & RAD50 homolog (S. cerevisiae) \\
\hline RAD5I & RAD5I homolog (S. cerevisiae) \\
\hline RAD5 IAPI & RAD5I associated protein I \\
\hline RAD5IB & RAD5I homolog B (S. cerevisiae) \\
\hline RAD5IC & RAD5I homolog C (S. cerevisiae) \\
\hline RAD5ID & RAD5I homolog D (S. cerevisiae) \\
\hline RAD52 & RAD52 homolog (S. cerevisiae) \\
\hline RAD54B & RAD54 homolog B (S. cerevisiae) \\
\hline RAD54L & RAD54-like (S. cerevisiae) \\
\hline RAD9A & RAD9 homolog A (S. pombe) \\
\hline$R B I$ & Retinoblastoma I \\
\hline RBBP8 & Retinoblastoma binding protein 8 \\
\hline RECQL & RecQ protein-like (DNA helicase QI-like) \\
\hline RECQL4 & RecQ protein-like 4 \\
\hline RECQL5 & RecQ protein-like 5 \\
\hline RET & Ret proto-oncogene \\
\hline RFCl & Replication factor C (activator I) I, I 45 kDa \\
\hline RFC2 & Replication factor C (activator I) 2, 40 kDa \\
\hline
\end{tabular}


Table 3 (Continued)

\begin{tabular}{|c|c|}
\hline Abbreviation & Gene name (alternative denominations) \\
\hline RFC4 & Replication factor C (activator I) 4, $37 \mathrm{kDa}$ \\
\hline RHBDF2 & Rhomboid 5 homolog 2 (Drosophila) \\
\hline RNFI46 & Ring finger protein I46 \\
\hline RNFI68 & Ring finger protein I68, E3 ubiquitin protein ligase \\
\hline RNF8 & Ring finger protein 8, E3 ubiquitin protein ligase \\
\hline RPAI & Replication protein $\mathrm{AI}, 70 \mathrm{kDa}$ \\
\hline RUNXI & Runt-related transcription factor I \\
\hline SDHAF2 & Succinate dehydrogenase complex assembly factor 2 \\
\hline SDHB & Succinate dehydrogenase complex, subunit B, iron sulfur (IP) \\
\hline SETBPI & SET binding protein I \\
\hline SETX & Senataxin \\
\hline SHPRH & SNF2 histone linker PHD RING helicase, E3 ubiquitin protein ligase \\
\hline SLX4 & SLX4 structure-specific endonuclease subunit homolog (S. cerevisiae) \\
\hline SMAD4 & SMAD family member 4 \\
\hline SMARCA4 & SWI/SNF-related, matrix-associated, actin-dependent regulator of chromatin, subfamily a, member 4 \\
\hline SMARCBI & SWI/SNF-related, matrix-associated, actin-dependent regulator of chromatin, subfamily b, member I \\
\hline SMARCEI & SWI/SNF-related, matrix-associated, actin-dependent regulator of chromatin, subfamily e, member I \\
\hline STKII & Serine/threonine kinase II \\
\hline SUFU & Suppressor of fused homolog (Drosophila) \\
\hline TCLIA & T-cell leukemia/lymphoma IA \\
\hline TELO2 & TEL2, telomere maintenance 2, homolog (S. cerevisiae) \\
\hline TERF2 & Telomeric repeat binding factor 2 \\
\hline TERT & Telomerase reverse transcriptase \\
\hline TLR2 & Toll-like receptor 2 \\
\hline TLR4 & Toll-like receptor 4 \\
\hline TMEM/27 & Transmembrane protein 127 \\
\hline TOPBPI & Topoisomerase (DNA) II binding protein I \\
\hline TP53 & Tumor protein p53 \\
\hline TP53BPI & Tumor protein $\mathrm{p} 53$ binding protein I \\
\hline TSCl & Tuberous sclerosis I \\
\hline TSC2 & Tuberous sclerosis 2 \\
\hline TSHR & Thyroid stimulating hormone receptor \\
\hline UBE2A & Ubiquitin-conjugating enzyme E2A \\
\hline UBE2B & Ubiquitin-conjugating enzyme E2B \\
\hline UBE2I & Ubiquitin-conjugating enzyme E2I \\
\hline UBE2V2 & Ubiquitin-conjugating enzyme E2 variant 2 \\
\hline UBE4B & Ubiquitination factor E4B \\
\hline UIMCI & Ubiquitin interaction motif containing I \\
\hline VHL & Von Hippel-Lindau tumor suppressor, E3 ubiquitin protein ligase \\
\hline WRN & Werner syndrome, RecQ helicase-like \\
\hline WTI & Wilms tumor I \\
\hline$X P A$ & Xeroderma pigmentosum, complementation group $\mathrm{A}$ \\
\hline$X P C$ & Xeroderma pigmentosum, complementation group $\mathrm{C}$ \\
\hline$X R C C I$ & X-ray repair complementing defective repair in Chinese hamster cells I \\
\hline$X R C C 2$ & X-ray repair complementing defective repair in Chinese hamster cells 2 \\
\hline$X R C C 3$ & X-ray repair complementing defective repair in Chinese hamster cells 3 \\
\hline$X R C C 4$ & X-ray repair complementing defective repair in Chinese hamster cells 4 \\
\hline$X R C C 5$ & X-ray repair complementing defective repair in Chinese hamster cells 5 \\
\hline$X R C C 6$ & X-ray repair complementing defective repair in Chinese hamster cells 6 \\
\hline ZNF350 & Zinc finger protein 350 \\
\hline ZNF365 & Zinc finger protein 365 \\
\hline
\end{tabular}


Table 4 Table of identified variants classified as likely pathogenic/pathogenic according to the ClinVar database

\begin{tabular}{|c|c|c|c|c|c|c|c|}
\hline Patient & Gene & Nucleotide & Protein & $\begin{array}{l}\text { ClinVar } \\
\text { classification }\end{array}$ & $\begin{array}{l}\text { Sex/age } \\
\text { primary }\end{array}$ & $\begin{array}{l}\text { Personal } \\
\text { history (age at } \\
\text { diagnosis) }\end{array}$ & Family history \\
\hline \multicolumn{8}{|c|}{ With SMN } \\
\hline OL0I38 & CHEK2 & c. $349 A>G$ & p.ArgII7Gly & Class 4-5 & Female/70 & Breast (7I) & 0 \\
\hline \multicolumn{8}{|c|}{ Without SMN } \\
\hline OL0I30 & RAD5ID & c. $345+2 T>G$ & - & Class 4 & Male/62 & 0 & Mother - gastric \\
\hline OL0132 & MLHI & c.390C>G & p.Tyrl30Ter & Class 5 & \multirow[t]{2}{*}{ Female/52 } & \multirow[t]{2}{*}{0} & \multirow{2}{*}{$\begin{array}{l}\text { Father - colon, father's } \\
\text { mother - brain }\end{array}$} \\
\hline & ATM & c.3849delA & p.Leu I283fs & Class 5 & & & \\
\hline $\mathrm{PCl77}$ & CHEK2 & c.II00delC & p.Thr367fs & Class 5 & Male/55 & 0 & 0 \\
\hline
\end{tabular}

Note: All variants are heterozygous.

Abbreviation: SMN, subsequent malignant neoplasm after pancreatic ductal adenocarcinoma (PDAC).

Table 5 List of identified variants of unknown significance

\begin{tabular}{|c|c|c|c|c|c|c|c|c|c|c|}
\hline Patient & Gene & Nucleotide & Protein & rs number & $\begin{array}{l}\text { EXaC } \\
\text { MAF }\end{array}$ & $\begin{array}{l}\text { ClinVarl } \\
\text { VarSome } \\
\text { classification }\end{array}$ & SIFT & PP2 & MA & $\begin{array}{l}\text { Damag. } \\
\text { acc. to } \geq 2 \\
\text { software }\end{array}$ \\
\hline \multicolumn{11}{|c|}{ With SMN } \\
\hline OL0I34 & $B L M$ & c. IIT>C & p.Val4Ala & rsI44706057 & 0.0017 & $\mathrm{I}-3 / 3$ & 0 & 0.132 & 2.14 & $\mathrm{Y}$ \\
\hline \multirow[t]{2}{*}{ OL0135 } & PTCHI & c.2597G $>A$ & p.Gly866Glu & NA & NA & $3 / 3$ & 0.08 & 0.999 & 2.31 & $\mathrm{Y}$ \\
\hline & ATM & c. $3208 \mathrm{G}>\mathrm{A}$ & p.Vall 070lle & NA & NA & $3 / 3$ & 0.35 & 0.026 & 2.135 & $\mathrm{~N}$ \\
\hline \multirow[t]{3}{*}{ OL0I36 } & PLA2G2A & c. $185 \mathrm{G}>\mathrm{A}$ & p.Arg62His & NA & $8.34 \mathrm{E}-05$ & $\mathrm{NA} / 3$ & 0.02 & 0.888 & 3.005 & $\mathrm{Y}$ \\
\hline & LRIGI & $c .2195 \mathrm{C}>\mathrm{T}$ & p.Pro732Leu & rs617463346 & 0.0022 & $\mathrm{NA} / 3$ & 0 & 0.991 & 1.975 & $\mathrm{Y}$ \\
\hline & RECQL5 & c. $|80| \mathrm{G}>\mathrm{A}$ & p.Val60IMet & NA & NA & $\mathrm{NA} / 3$ & 0.3 & 0.04 & 1.905 & $\mathrm{~N}$ \\
\hline \multirow[t]{2}{*}{ OLOI38 } & PREX2 & c.Cl672G & p.Pro558Ala & rs19954I834 & 0.0001 & $\mathrm{NA} / 3$ & 0.15 & 0.145 & 0.46 & $\mathrm{~N}$ \\
\hline & PARPI & c.C659T & p.Ala220Val & rsI39232092 & 0.0006 & $\mathrm{NA} / 3$ & 0.15 & 0.003 & 1.155 & $\mathrm{~N}$ \\
\hline \multicolumn{11}{|c|}{ Without SMN } \\
\hline \multirow[t]{2}{*}{ OL004I } & $B U B / B$ & c. $1042 \mathrm{G}>\mathrm{A}$ & p.Ala348Thr & NA & $8.24 \mathrm{E}-06$ & $\mathrm{NA} / 3$ & 0.33 & 0.85 & 2.175 & $\mathrm{~N}$ \\
\hline & MREIIA & c.CI475A & p.Ala492Asp & rs61749249 & 0.0034 & $1-3 / 3$ & 0.43 & 0.754 & 1.735 & $\mathrm{~N}$ \\
\hline OLOI30 & XRCCI & c.632A $>G$ & p.Tyr2 I ICys & NA & $1.74 \mathrm{E}-05$ & $\mathrm{NA} / 3$ & 0.15 & 0.998 & 2.175 & $\mathrm{Y}$ \\
\hline OL0I3I & 0 & & & & & & & & & \\
\hline \multirow[t]{2}{*}{ OL0132 } & GRB7 & c. $1439 \mathrm{~T}>\mathrm{C}$ & p.Val480Ala & rs|4337293I & 0.0004 & $\mathrm{NA} / 3$ & 0 & 0.848 & 3.07 & $\mathrm{Y}$ \\
\hline & RAD9A & $c .215 \mathrm{G}>\mathrm{A}$ & p.Arg72His & rs37729983I & I.65E-05 & $\mathrm{NA} / 3$ & 0.58 & 0.019 & 1.2 & $\mathrm{~N}$ \\
\hline \multirow[t]{2}{*}{ OL0I33 } & EXT2 & c. $1859 \mathrm{C}>\mathrm{T}$ & p.Thr620Met & rs|38495222 & 0.0006 & $2-3 / 3$ & 0.02 & 0.999 & 2.24 & $\mathrm{Y}$ \\
\hline & $M L \mathrm{H3}^{a}$ & c. $328 I-I G>C$ & - & NA & NA & $\mathrm{NA} / 3$ & - & - & - & - \\
\hline \multirow[t]{3}{*}{ OL0I37 } & PREX2 & c. $2167 A>G$ & p.Asn723Asp & NA & I.65E-05 & $\mathrm{NA} / 3$ & 0.03 & 0.614 & 1.63 & $\mathrm{~N}$ \\
\hline & HELQ & c. $1418 \mathrm{G}>\mathrm{A}$ & p.Arg473His & NA & $2.48 \mathrm{E}-05$ & NA/3 & 0 & I & 4.545 & $Y$ \\
\hline & RFC4 & c. $908 \mathrm{C}>\mathrm{T}$ & p.Ala303Val & rs|44238574 & $9.07 \mathrm{E}-05$ & $\mathrm{NA} / 3$ & 0.44 & 0.027 & 1.235 & $\mathrm{~N}$ \\
\hline \multirow[t]{2}{*}{ OL0139 } & RHBDF2 & c. $940 \mathrm{G}>\mathrm{A}$ & p.Ala3I4Thr & rs|40433374 & 0.0008 & NA/3 & 0.33 & 0.952 & 1.78 & $\mathrm{~N}$ \\
\hline & MDM4 & c. $1162 C>G$ & p.Pro388Ala & rs61754765 & 0.0006 & $\mathrm{NA} / 3$ & 0.92 & 0.997 & 1.1 & $\mathrm{~N}$ \\
\hline \multirow[t]{2}{*}{ OLOI40 } & FANCM & c.3407T $>C$ & p.Leu I I36Ser & NA & I.65E-05 & $\mathrm{NA} / 3$ & 0.01 & 0.963 & 1.905 & $\mathrm{Y}$ \\
\hline & POLE & c. $160 I T>C$ & p.Leu534Pro & NA & NA & $\mathrm{NA} / 3$ & 0 & 0.991 & 3.565 & $Y$ \\
\hline OLOI4I & 0 & & & & & & & & & \\
\hline \multirow[t]{2}{*}{ OL0I42 } & RAD54L & c. $1817 \mathrm{G}>\mathrm{A}$ & p.Arg606GIn & rs37457494I & $2.47 \mathrm{E}-05$ & $\mathrm{NA} / 3$ & 0 & $\mathbf{I}$ & 4.735 & $Y$ \\
\hline & POLDI & $c .2116 C>G$ & p.Pro706Ala & NA & NA & $3 / 3$ & 0.01 & 0.733 & 2.41 & $\mathrm{Y}$ \\
\hline \multirow[t]{2}{*}{ OL0I44 } & CWFI9L2 & c. $2240 A>C$ & p.Lys747Ther & NA & $\mathrm{NA}$ & $\mathrm{NA} / 3$ & 0.08 & 0.697 & 1.915 & $\mathrm{~N}$ \\
\hline & SETX & $c .967 A>G$ & p.Ser323Gly & NA & I.65E-05 & $\mathrm{NA} / 3$ & 0 & 0.994 & 0.975 & $Y$ \\
\hline OL0I57 & TP53BPI & c. $2226 \mathrm{~A}>\mathrm{T}$ & p.Glu742Asp & rsI50423877 & 0.0004 & $\mathrm{NA} / 3$ & 0.48 & 0.987 & 0.46 & $\mathrm{~N}$ \\
\hline $\mathrm{PCl} 77$ & 0 & & & & & & & & & \\
\hline \multirow[t]{2}{*}{$\mathrm{PClI} 5$} & PTCHI & c.3376G>A & p.Vall I26lle & rs|47025073 & 0.0005 & $3 / 3$ & 0.26 & 0.927 & 1.77 & $\mathrm{~N}$ \\
\hline & NCAMI & c. $|48| C>A$ & p.Thr494Asn & NA & NA & $\mathrm{NA} / 3$ & 0.01 & 0.347 & $\mathrm{NA}$ & $\mathrm{N}$ \\
\hline $\mathrm{PCl} 39$ & 0 & & & & & & & & & \\
\hline \multirow[t]{3}{*}{ PCOII } & BRCAI & c.3929C>A & p.ThrI3IOLys & rs80357257 & $8.24 \mathrm{E}-06$ & $1-3 / 3$ & 0.01 & 0.787 & 1.895 & $\mathrm{~N}$ \\
\hline & AURKA & c. $1028 \mathrm{G}>\mathrm{A}$ & p.Arg343Gln & rs200I8I472 & 0.0002 & $N A / 3$ & 0.04 & 0.027 & 0.71 & $\mathrm{~N}$ \\
\hline & EXOI & c.820G $>A$ & p.Gly274Arg & rs|49397534 & 0.0021 & $N A / 3$ & 0.16 & 0.999 & 1.295 & $\mathrm{~N}$ \\
\hline
\end{tabular}

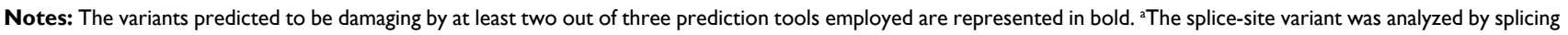
prediction software spidex with a score -25.6359 , suggesting that it is the damaging variant.

Abbreviation: NA, not applicable. 
cancers, including PDAC survivors. ${ }^{17}$ The same trend has also been confirmed in the Czech population..$^{18}$ A higher age at the time of the primary PDAC diagnosis was the only remarkable difference between five-year survivors with SMNs and those without SMNs. The incidence of cancer increases with age, and, consequently, older survivors have a higher risk of SMNs than younger survivors. All patients with a manifestation of SMN received adjuvant chemotherapy consisting of antimetabolites gemcitabine or 5-fluorouracil. Although patients who undergo chemotherapy are generally considered to be at a higher risk of SMN, an increased risk of SMNs after the use of these antimetabolites has not been reported to date.

Therefore, it seems that a higher age at the time of the PDAC diagnosis and a long-term survival after a surgical and chemotherapy treatment may be regarded as risk factors for SMNs, and that such patients should be diagnostically followed.

The NGS analysis revealed five clearly pathogenic variants in four patients from the long-term PDAC survivors subgroup (25\%). This frequency was higher than for the other group of 96 unselected PDAC patients, ${ }^{19}$ which was $13.5 \%$ identified with a panel of 22 genes, but we are aware of the small number of patients analyzed in our study. A recent study by Yurgelun et a $\mathrm{l}^{20}$ identified 28 carriers of germline pathogenic or likely pathogenic mutations in double-strand DNA damage repair genes in 289 patients (9.7\%) with resected PDAC. Interestingly, the authors demonstrated that the germline mutations carriers had superior overall survival (HR $0.54 ; P=0.05$ ). This indicates that mutations in cancerpredisposing genes increase the risk of prognostically beneficial PDAC; therefore, it might be expected that an increased proportion of mutation carriers should also be found among the long-term PDAC survivors. Unfortunately, the genetic aberrations discovered do not currently seem to be of any clinical relevance with regard to potential therapeutic options.

Considering the small number of long-term survivors, the frequency of pathogenic variants in the group of patients who developed SMNs (25\%) and in the group who did not (19\%) was comparable. These results suggest that SMN development may be due to a combined effect of variants with low penetrance or may be caused by a combination of genetic and/or nongenetic risk factors. On the other hand, the presence of germline mutations did not dramatically influence risk and prognosis of SMN.

The patient with PDAC at 70 years old and subsequent breast cancer at 71 was identified to harbor a pathogenic missense CHEK2 variant (c.349A>G, p.Arg117Gly). Numerous studies and meta-analyses have shown that mutations in the CHEK2 gene are clearly associated with increased breast cancer risk and also with the development of other solid or hematologic tumors. ${ }^{21}$ We failed to find a significant association of CHEK2 germline variants with unselected PDAC cases in our previous study; however, only selected portions of $C H E K 2$ coding sequence were analyzed. ${ }^{22}$ Since then, germline CHEK2 mutations have been identified in several studies in patients with PDAC; ${ }^{19,20,23,24}$ however, a consensual evaluation of $C H E K 2$ germline variants in PDAC remains to be established.

In a subgroup of 16 long-term PDAC survivors without SMN development, we identified 2 PDAC patients with pathogenic variants in cancer predisposition genes and a positive family history. MLH1 is a Lynch syndrome predisposition gene ${ }^{25}$ and can explain the colorectal cancer in the patient's father. $R A D 51 D$ is an ovarian cancer predisposition gene, ${ }^{26}$ but was never associated with gastric cancer. These data indicate that germline mutations in cancer predisposition genes are associated with a wider range of phenotypes than previously suggested.

The evaluation of potentially pathogenic missense germline variants in candidate genes requires further analysis in larger groups of PDAC patients, as well as functional studies, because in silico predictions are suitable for variant prioritization for such analyses, but are not devoted to final variant classification.

The present study, therefore, poses new questions regarding the role of genetic alterations in the development of PDAC and subsequent SMNs in patients, and regarding the modification of the clinical course of the disease. The variants identified in the present study must be verified by further investigations, also in regard to the functional impact. However, this is the first study of genetic alterations in SMNs in PDAC patients and the largest epidemiologic retrospective analysis of SMNs after PDAC treatment in Central Europe.

\section{Conclusion}

In our cohort, $27 \%$ of five-year PDAC survivors went on to develop SMNs. An intensive follow-up can identify the second primary neoplasms early, at a curable stage. SMN risk factors include a longer survival and a higher age at the time of PDAC diagnosis. Genetic analysis has confirmed the role of pathogenic mutations in pancreatic and other cancers' predisposition genes in long-term surviving PDAC patients; nevertheless, the frequency did not differ in the subgroups with and without SMN development. If the performance status of these patients allows and a second primary tumor 
has a favorable prognosis, subsequent surgery should be performed.

\section{Acknowledgments}

This work was supported by the Ministry of Health of the Czech Republic (grant no. 16-28375A to BM-D, 16-29959A to ZK, and 16-31314A to PS), and the Czech Ministry of Education (no. NPU I LO1304, LO1503, and RVO: 61989592).

\section{Disclosure}

A grant from Palacky University was awarded to $\mathrm{TZ}$ (IGA_LF_2018_010), Charles University Projects (UNCE/ MED/006) was awarded to PSo, and PROGRES grants (Q28/ LF1 and SVV 260367) were awarded to PZ, KL, and MB. The authors report no other conflicts of interest in this work.

\section{References}

1. Grundmann RT, Meyer F. Second primary malignancy among cancer survivors - epidemiology, prognosis and clinical relevance. Zentralbl Chir. 2012;137(6):565-574.

2. Lovecek M, Skalicky P, Klos D, et al. Long-term survival after resections for pancreatic ductal adenocarcinoma. Single centre study. Biomed Pap Med Fac Univ Palacky Olomouc Czech Repub. 2016;160(2): 280-286.

3. Ng AK, Travis LB. Subsequent malignant neoplasms in cancer survivors. Cancer J. 2008;14(6):429-434.

4. Teepen JC, van Leeuwen FE, Tissing WJ, et al. Long-term risk of subsequent malignant neoplasms after treatment of childhood cancer in the DCOG LATER study cohort: role of chemotherapy. J Clin Oncol. 2017;35(20):2288-2298.

5. Minni F, Casadei R, Marrano N, et al. Second tumours in patients with malignant neoplasms of the digestive apparatus. A retrospective study on 2406 cases. Ann Ital Chir. 2005;76(5):467-472.

6. Jacobs NL, Que FG, Miller RC, Vege SS, Farnell MB, Jatoi A. Cumulative morbidity and late mortality in long-term survivors of exocrine pancreas cancer. J Gastrointest Cancer. 2009;40(1-2):46-50.

7. Gerdes B, Ziegler A, Ramaswamy A, Wild A, Langer P, Bartsch DK. Multiple primaries in pancreatic cancer patients: indicator of a genetic predisposition? Int J Epidemiol. 2000;29(6):999-1003.

8. Riall TS, Stager VM, Nealon WH, et al. Incidence of additional primary cancers in patients with invasive intraductal papillary mucinous neoplasms and sporadic pancreatic adenocarcinomas. J Am Coll Surg. 2007;204(5):803-813.

9. Chen KT, Devarajan K, Hoffman JP. Morbidity among long-term survivors after pancreatoduodenectomy for pancreatic adenocarcinoma. Ann Surg Oncol. 2015;22(4):1185-1189.
10. Sobin LH, Gospodarowicz M, Wittekind Ch, eds. TNM Classification of Malignant Tumours. 7th ed. New York, Hoboken: Wiley-Blackwell; 2009

11. Sugimura H, Caporaso NE, Shaw GL, et al. Human debrisoquine hydroxylase gene polymorphisms in cancer patients and controls. Carcinogenesis. 1990;11(9):1527-1530.

12. Soukupova J, Zemankova P, Lhotova K, et al. Validation of CZECANCA (CZEch CAncer paNel for Clinical Application) for targeted NGSbased analysis of hereditary cancer syndromes. PLoS One. 2018;13(4): e0195761.

13. Wang K, Li M, Hakonarson H. ANNOVAR: functional annotation of genetic variants from high-throughput sequencing data. Nucleic Acids Res. 2010;38(16):e164.

14. AIRTUM Working Group. Italian cancer figures, report 2013: Multiple tumours. Epidemiol Prev. 2013;37(4-5 Suppl 1):1-152.

15. Cancer Incidence in the Czech Republic, UZIS, Czech Republic, 2011. Available from: www.uzis.cz/en/publications/cancer-incidence-2011. Accessed February 5, 2018

16. Hackert T, Tjaden C, Müller S, et al. Extrapancreatic malignancies in patients with pancreatic cancer: epidemiology and clinical consequences. Pancreas. 2012;41(2):212-217.

17. Schnelldorfer T, Ware AL, Sarr MG, et al. Long-term survival after pancreatoduodenectomy for pancreatic adenocarcinoma: is cure possible? Ann Surg. 2008;247(3):456-462.

18. Pavlík T, Májek O, Büchler T, et al. Trends in stage-specific populationbased survival of cancer patients in the Czech Republic in the period 2000-2008. Cancer Epidemiol. 2014;38(1):28-34.

19. Hu C, Hart SN, Bamlet WR, et al. Prevalence of pathogenic mutations in cancer predisposition genes among pancreatic cancer patients. Cancer Epidemiol Biomarkers Prev. 2016;25(1):207-211.

20. Yurgelun MB, Chittenden AB, Morales-Oyarvide V, et al. Germline cancer susceptibility gene variants, somatic second hits, and survival outcomes in patients with resected pancreatic cancer. Genet Med. Epub 2018 July 2.

21. Havranek O, Kleiblova P, Hojny J, et al. Association of germline CHEK2 gene variants with risk and prognosis of non-hodgkin lymphoma. PLoS One. 2015;10(10):e0140819.

22. Mohelnikova-Duchonova B, Havranek O, Hlavata I, et al. CHEK2 gene alterations in the forkhead-associated domain, 1100delC and del5395 do not modify the risk of sporadic pancreatic cancer. Cancer Epidemiol. 2010;34(5):656-658.

23. Chaffee KG, Oberg AL, McWilliams RR, et al. Prevalence of germline mutations in cancer genes among pancreatic cancer patients with a positive family history. Genet Med. 2018;20(1):119-127.

24. Young EL, Thompson BA, Neklason DW, et al. Pancreatic cancer as a sentinel for hereditary cancer predisposition. BMC Cancer. 2018;18(1):697.

25. Nallamilli BRR, Hegde M. Genetic testing for hereditary nonpolyposis colorectal cancer (HNPCC). CurrProtoc Hum Genet. 2017;94(1): 10.12.1-10.12.23.

26. Eoh KJ, Kim JE, Park HS, et al. Detection of germline mutations in patients with epithelial ovarian cancer using multi-gene panels: beyond BRCA1/2. Cancer Res Treat. 2018;50(3):917-925.
Cancer Management and Research

\section{Publish your work in this journal}

Cancer Management and Research is an international, peer-reviewed open access journal focusing on cancer research and the optimal use of preventative and integrated treatment interventions to achieve improved outcomes, enhanced survival and quality of life for the cancer patient. The manuscript management system is completely online and includes 